\title{
Pour une meilleure compréhension de l'estime de soi : liens entre la perception de soi et les indicateurs sociométriques
}

\section{Philippe A. Genoud}

Volume 11, numéro 1, 2008

URI : https://id.erudit.org/iderudit/1017508ar

DOI : https://doi.org/10.7202/1017508ar

Aller au sommaire du numéro

Éditeur(s)

Faculté d'éducation, Université de Sherbrooke

ISSN

1911-8805 (numérique)

Découvrir la revue

Citer cet article

Genoud, P. A. (2008). Pour une meilleure compréhension de l'estime de soi : liens entre la perception de soi et les indicateurs sociométriques. Nouveaux cahiers de la recherche en éducation, 11(1), 35-47.

https://doi.org/10.7202/1017508ar
Résumé de l'article

L'objectif de cette recherche est d'explorer les liens entre l'estime de soi et les différents indicateurs sociométriques. Nous avons utilisé le questionnaire Reflet permettant ainsi de prendre en compte les perceptions de l'élève quant à ses relations dans la classe. Les analyses effectuées, portant sur un échantillon de 263 élèves, indiquent que le statut sociométrique imaginé apporte un élément supplémentaire à la compréhension de l'estime de soi dans le domaine social. Si les sympathies réciproques entre camarades dans la classe constituent un facteur important dans la construction de l'image de soi, nos résultats soulignent le caractère prédominant de la perception qu'a l'élève des relations qu'il entretient avec ses pairs. 


\title{
Pour une meilleure compréhension de l'estime de soi : liens entre la perception de soi et les indicateurs sociométriques
}

\author{
Philippe A. Genoud \\ Université de Fribourg
}

\begin{abstract}
Résumé
L'objectif de cette recherche est d'explorer les liens entre l'estime de soi et les différents indicateurs sociométriques. Nous avons utilisé le questionnaire Reflet permettant ainsi de prendre en compte les perceptions de l'élève quant à ses relations dans la classe. Les analyses effectuées, portant sur un échantillon de 263 élèves, indiquent que le statut sociométrique imaginé apporte un élément supplémentaire à la compréhension de l'estime de soi dans le domaine social. Si les sympathies réciproques entre camarades dans la classe constituent un facteur important dans la construction de l'image de soi, nos résultats soulignent le caractère prédominant de la perception qu'a l'élève des relations qu'il entretient avec ses pairs.
\end{abstract}

\begin{abstract}
The objective of this study was to explore the connections between self-esteem and the various sociometric indicators. We used both the basic questionnaire and its «reflective» variant, allowing for taking into account pupils' own perceptions about their classroom relationships. Analyses were done on a sample of 263 pupils. They suggest that imagined sociometric status adds a supplementary factor to the understanding of self-esteem in the social sphere. While affinities between classmates are a significant factor in self-image construction, our findings highlight the predominance of the pupil's own perception of his or her relationships with peers.
\end{abstract}




\section{Introduction}

L'organisation et la structure des interactions sociales à l'intérieur d'un groupe dépendent des affinités entre les membres qui le constituent. Dans la classe tout particulièrement, le groupe est généralement clairement défini (connaissance de chacun des membres qui en font - ou pas - partie) et le temps passé dans des activités communes (en classe comme à la récréation) permet aux élèves de se forger rapidement une opinion sur leurs pairs.

La place que prend chaque élève au sein du groupe revêt une importance toute particulière tant pour le développement social que pour son impact sur le bien-être et les apprentissages scolaires. Toutefois, au-delà du statut réel de l'enfant comparé à ses pairs, sa propre perception de sa situation peut avoir une portée encore plus grande.

Dans cet article, après avoir discuté des différentes façons d'envisager la mesure sociométrique en classe et après avoir mentionné les problèmes éthiques qui peuvent en découler, nous examinerons les liens privilégiés entre l'estime de soi, le statut social au sein de la classe ainsi que la représentation qu'a l'élève de son propre statut. Sur la base d'une recherche empirique, nous donnerons des pistes permettant de mieux comprendre l'articulation de ces concepts.

\section{Problématique}

Grâce aux premiers développements de la sociométrie (Moreno, 1945), les enseignants et les chercheurs ont disposé d'un outil pour obtenir un cliché (appelé sociogramme) de la configuration des interactions à l'intérieur de la classe. On trouve dans la littérature scientifique nombre de publications utilisant cet outil, et ceci jusqu'à la fin des années 1960 où il est très en vogue. Toujours utilisé par les praticiens aujourd'hui, on le repère tout de même dans un certain nombre de recherches, mais de manière moins fréquente.

La méthode de base constituant à demander à chaque membre du groupe de nommer les trois camarades préférés ainsi que les trois les moins appréciés a évolué au fil des années et certains auteurs ont proposé d'autres démarches. Northway (1964) ainsi que Remmers (1963) par exemple proposent de laisser le nombre de nominations libre afin d'éviter de falsifier la spontanéité du sujet et de créer ainsi un biais dans les résultats. Bastin (1970) présente quant à lui une méthode ordinale (classement de tous les membres du groupe dans un ordre croissant de préférence) et une méthode utilisant des échelles d'estimation (évaluation de chaque pair sur une échelle de Likert). Cette dernière méthode permet également d'identifier de manière précise les élèves rejetés et conserve une bonne stabilité dans le temps (Asher et Dodge, 1986). De plus, en calculant la moyenne des scores donnés par un élève à ses camarades, on obtient un indice supplémentaire appelé expansivité affective.

Parmi les critères de choix (autrement dit la nature de l'activité pour laquelle chacun est invité à désigner ses préférences) apparaissent également des propositions variées. Du critère de base («Quel camarade trouves-tu sympathique?») aux critères plus spécifiques («Avec quels camarades souhaiterais-tu travailler en classe, jouer à la récréation, inviter pour ton anniversaire?, etc.»), les situations devraient inciter les répondants à choisir leurs pairs dans des situations authentiques nécessitant un engagement plus profond (Moreno, 1970), et éviter ainsi les situations ressenties comme fictives (par exemple: «Avec quels camarades voudrais-tu vivre sur une île déserte?»). 
Avec de jeunes élèves (7-8 ans), la différence entre les critères de choix utilisés apparaît comme minime. Par exemple, Toesca (1972) met en évidence de fortes corrélations dans les scores des élèves en utilisant des critères variés. Progressivement avec l'âge, les enfants distinguent plus facilement les situations et varieront plus fortement leurs évaluations en fonction du critère proposé (Frederickson et Furnham, 1998).

Plusieurs chercheurs (Coie, Dodge et Coppotelli, 1982; Ollendick, Weist, Borden et Greene, 1992) soulignent l'importance de différencier les sujets non seulement en fonction de leur statut sociométrique dans la classe, mais aussi selon des typologies permettant de différencier par exemple les individus négligés, rejetés et controversés. Cette distinction se base en fonction des scores sociométriques obtenus et du nombre de liens réciproques, ceci relativement aux autres camarades de la classe particulière. Ainsi, le type d'un élève dépendra bien évidemment de son statut sociométrique, mais aussi des statuts obtenus par ses pairs dans la classe. Cette standardisation permet également la comparaison entre des classes ayant un nombre d'élèves très différent.

Une autre évolution importante dans l'utilisation des démarches sociométriques concerne l'ajout d'une demande dite reflet à chacun des membres du groupe (Tagiuri, Kogan et Bruner, 1955), généralement sous la forme suivante: «Qui, parmi tes camarades, te trouve sympathique?». La prise en considération de la perception sociale (chacun essaie de deviner le plus exactement possible les choix des autres) contribue à une meilleure compréhension des interactions. En fonction des préférences attendues se joue également la dynamique relationnelle à l'intérieur de la classe. On peut ainsi constater des différences entre les élèves avec d'autres indicateurs, tels que les indices de lucidité, de transparence ou le statut sociométrique imaginé.

Avant de se pencher sur les recherches qui ont mis en évidence des liens entre les comportements des élèves, leur statut sociométrique et les répercussions sur l'estime de soi, il convient tout d'abord de soulever ici deux points essentiels concernant les mesures sociométriques : l'évaluation de la popularité ainsi que le questionnement éthique relatif à la démarche.

\subsection{Sociométrie et popularité : deux concepts différents}

$\mathrm{Si}$, dans la majorité des recherches, on voit fréquemment apparaître le terme populaire pour désigner les élèves ayant obtenu dans le groupe les scores les plus élevés à l'analyse sociométrique, plusieurs auteurs ont enjoint leurs collègues de différencier le statut sociométrique du concept de popularité. En effet, Parkhurst et Hopmeyer (1998) mentionnent que les élèves étant en moyenne beaucoup appréciés par leurs pairs ne sont pas forcément ceux qui sont considérés comme les plus populaires dans la classe. Selon ces auteurs, la popularité est plus liée aux comportements de dominance dans le groupe et les élèves qui sont désignés par leurs camarades comme populaires sont parfois controversés. La faiblesse des corrélations trouvées entre les deux concepts est également soulignée par Babad (2001), qui parle alors d'attractivité pour caractériser cette notion de statut sociométrique.

Reprenant cette problématique dans une publication plus récente encore, Košir et Pečjak (2005) observent une différence plus marquée entre le statut sociométrique et la popularité perçue par les pairs chez les adolescents. En effet, si les deux évaluations sont moyennement corrélées à l'âge de 11 ans, elles apparaissent comme indépendantes chez les élèves de 17 ans. Avec une méthodologie similaire, LaFontana et Cillessen (1999) relèvent non seulement que les filles identifient 
de manière plus précise que les garçons les élèves populaires de la classe, mais qu'il n'y a pas forcément de consensus sur cette évaluation de la popularité. Ces différentes recherches étayent donc la nécessité de distinguer la popularité perçue du statut sociométrique désignant les élèves les plus appréciés.

\subsection{Problèmes éthiques soulevés}

L'utilisation d'une démarche consistant à demander en particulier aux élèves de désigner nominativement (même de manière confidentielle) les camarades les moins appréciés a suscité un questionnement d'ordre éthique. Alors que Toesca (1972) parle d'un risque de cassure dans la classe, d'autres auteurs, dont Asher et Dodge (1986), privilégient des échelles d'estimation (évaluation de chaque pair) pour diminuer les effets potentiellement nuisibles d'une catégorisation négative de certains enfants.

Plusieurs recherches se sont alors penchées sur l'impact que pourrait avoir le questionnaire sociométrique sur les interactions entre les élèves et sur la dynamique dans la classe. Examinant les comportements des élèves dans leurs relations avec les autres camarades en général et avec les pairs désignés (positivement et négativement) - et mesurant également l'humeur et le degré de solitude ressentie -, Bell-Dolan, Foster et Sikora (1989) ne relèvent aucune influence de l'utilisation d'un questionnaire sociométrique dans des petits groupes. Une autre recherche (Hayvren et Hymel, 1984) compare, quant à elle, les différences éventuelles selon la méthodologie utilisée (nominations positives et négatives vs échelles d'estimation). Les analyses effectuées indiquent que le test sociométrique ne modifie en rien les comportements observés avant la passation, que ce soit dans un délai très court ou plus long. Par conséquent, alors que le questionnement éthique reste légitime, l'éventualité de voir apparaître des répercussions négatives dans les classes semble inexistante.

\subsection{Lien entre comportement et statut de l'élève}

Dans les multiples tentatives visant à comprendre pourquoi un enfant est apprécié de ses camarades alors qu'un autre ne l'est pas, les chercheurs ont, de manière constante, examiné les comportements et les compétences des élèves dans leurs interactions sociales (McArdle, O'Brien, Macmillan et Kolvin, 2000; Newcomb, Bukowski et Pattee, 1993). Putallaz (1983), par exemple, obtient une prédiction relativement précise du statut sociométrique d'un enfant en se basant sur la manière dont il entre en interaction avec un pair inconnu plusieurs mois avant le début de la scolarité. Par contre, lorsqu'il s'agit de compétences hypothétiques de résolution de problèmes relationnels (on demande à l'enfant ce qu'il ferait dans une situation décrite), les différences entre les élèves appréciés et les autres s'avèrent beaucoup moins importantes (Brochin et Wasik, 1992).

Malgré les critiques relatives au caractère artificiel et décontextualisé du questionnaire sociométrique (Northway, 1964), on constate, dans les conclusions de nombreuses publications, que ce sont bien les comportements des élèves qui permettent de discriminer leurs différents statuts sociométriques mesurés (Coie et Dodge, 1988; Eisenberg, Pidada et Liew, 2001). Des recherches connexes ont également pu montrer des liens entre les mesures sociométriques et les interactions observées dans des tâches à effectuer avec les parents (Franz et Gross, 2001) ainsi qu'avec l'expression des émotions (Hubbard, 2001). Il y a donc une évidence empirique des liens entre les comportements relationnels des élèves et les scores sociométriques qui leur sont attribués par leurs pairs. 


\subsection{Répercussions sur l'estime de soi}

Alors que les premières théories concernant les déterminants de l'estime de soi défendaient l'idée que cette dernière se forme à partir de nos échecs et de nos réussites, d'autres auteurs ont avancé progressivement l'idée qu'il s'agit d'une construction sociale, et que le regard des autres tel que perçu par le sujet - est à même d'améliorer ou d'altérer cette image de soi-même (Harter, 1998). Cette influence directe du regard d'autrui n'est actuellement plus remise en question dans la définition de l'estime de soi (Gilly, 1999; L'Ecuyer, 1994; Rosenberg, Schooler et Schoenbach, 1989). Pour André et Lelord (2007), il existe quatre sources principales de jugements dont dépend l'estime de soi d'un enfant: ses parents, ses enseignants, ses camarades de classe et ses amis. Toutes n'ont pas forcément le même poids et, si l'importance des parents est plus forte chez les jeunes enfants, elle diminue progressivement avec l'âge au profit des pairs.

Sachant que les sujets ont généralement conscience des acceptations et des rejets par leurs camarades et donc une perception sociale réaliste de leur statut dans la classe (Tagiuri, 1952), il n'est pas étonnant alors de voir des recherches mettant en lien perception de soi et typologie sociométrique (Boivin et Begin, 1989; Boivin, Vitaro et Gagnon, 1992; Bolognini et Plancherel, 1998; Rudolph, Hammen et Burge, 1995). De manière encore plus détaillée, Bishop et Inderbitzen (1995) différencient l'impact du statut sociométrique des liens réciproques dans la classe. Selon ces auteurs, les élèves possédant au moins une relation réciproque dans la classe ont une estime de soi plus élevée que ceux n'en ayant aucune, indépendamment de l'acceptation globale des camarades.

Dans cette recherche, nous visons à mieux comprendre les liens entre certains aspects interpersonnels (mesurés avec les outils sociométriques) et intrapersonnels, à savoir l'estime de soi. Plus concrètement, à l'aide d'une démarche principalement exploratoire, nous analyserons de quelle manière s'articulent les indicateurs sociométriques dans la construction de l'estime de soi de l'élève, tant avec des statistiques corrélationnelles qu'avec l'utilisation de la typologie proposée par Coie, Dodge et Coppotelli (1982). Nous examinerons également les liens entre la présence de relations réciproques chez un élève et son estime de soi. L'importance de l'expansivité affective (réelle et perçue) fera également l'objet d'une analyse détaillée. Finalement - et de manière plus secondaire -, nous formulons l'hypothèse que le statut sociométrique imaginé (mesure issue du questionnaire sociométrique Reflet) apporte une contribution supplémentaire significative à l'explication de l'estime de soi.

\section{Méthode}

\subsection{Participants}

L'échantillon est constitué de 263 élèves issus de 13 classes primaires du canton de Fribourg (Suisse). Les participants ont un âge compris entre 11 et 14 ans (moyenne d'âge: 11,9 ans). La répartition selon le genre est très équilibrée avec $51 \%$ de garçons et $49 \%$ de filles. Parmi les participants, $81 \%$ possèdent la nationalité suisse alors que le pourcentage restant (19\%) provient principalement de pays européens. 


\subsection{Procédure et mesures}

Les participants ont répondu à trois questionnaires successivement, après une brève introduction sur les objectifs généraux de la recherche. Les participants ont été invités à répondre de manière individuelle et les règles de confidentialité ont été présentées, à savoir que ni les parents, ni les enseignants ne pourraient avoir accès aux données individuelles concernant les élèves, et qu'aucun résultat par classe ne serait disponible. Le chercheur a été présent dans chacune des classes lors de la passation et s'est assuré que cette dernière se passe dans de bonnes conditions (travail individuel dans le calme).

Le SPP, version francophone - Il existe dans la documentation plusieurs validations francophones du Self-Perception Profile de Harter (1982) (Boivin, Vitaro et Gagnon, 1992; Worth Gavin et Herry, 1996). Nous avons opté pour celle proposée par Pierrehumbert, Plancherel et JankechCaretta (1987) puisque le contexte de validation est similaire à celui de notre recherche et que ces auteurs conservent l'ensemble des dimensions du questionnaire de base. Constitué de 30 items, cet outil francophone évalue, selon 6 échelles (ou notes partielles), l'estime de soi du répondant dans les domaines suivants: compétence à l'école (aspects scolaires et cognitifs), compétence sociale (relations avec les pairs), compétence physique (liée principalement aux activités sportives), apparence physique (degré de satisfaction quant à la taille, au poids, etc.), conduite (satisfaction face à son propre comportement) et sentiment de valeur propre (questions sur l'auto-satisfaction générale, formulées de manière non contextualisée). La consistance interne (alpha de Cronbach) calculée sur les 6 dimensions est comprise entre 0,64 et $0,77(\mathrm{~m}=0,71)$; pour notre échantillon, ces valeurs sont manifestement plus élevées puisque les alphas se situent entre 0,72 et $0,91(\mathrm{~m}=$ 0,83), bien que l'échantillon semble tout à fait comparable.

Les matrices sociométriques - Afin de mesurer différents indices liés à la sociométrie, nous avons présenté aux élèves le questionnaire de base ( «Voici les prénoms de tes camarades de classe, pour chacun, indique si tu le/la trouves sympathique.»), avec des échelles d'estimation de Likert à 5 positions. Une fois cette première partie terminée, nous avons distribué le questionnaire reflet (échelles de Likert à 5 positions également) comprenant la consigne suivante: «Voici les prénoms de tes camarades de classe, pour chacun, indique si tu penses qu'il/elle te trouve sympathique (essaie de deviner comment tes camarades t'ont évalué)».

Les données recueillies à l'aide de ces deux questionnaires sociométriques ont été organisées sous forme de matrices et neuf indices ont pu être calculés.

1. Statut sociométrique réel: indique si l'élève est apprécié (versus rejeté) par ses camarades (moyennes des évaluations des pairs de sa classe).

2. Statut sociométrique imaginé: indique si l'élève pense être apprécié par ses camarades (moyenne des scores sur la matrice Reflet).

3. Lucidité: indique si l'élève est conscient de (arrive à deviner) ce que pensent ses camarades à son sujet.

4. Expansivité affective réelle: indique le degré auquel l'élève apprécie en moyenne ses camarades (moyenne des évaluations données à ses pairs). 
5. Expansivité affective perçue: indique si l'élève est perçu par ses camarades comme appréciant ou non ces derniers.

6. Transparence: indique si, dans la classe, on devine facilement ce que l'élève pense de ses camarades.

7. Réciprocité: indique si un élève apprécie ses camarades en fonction (ou non) du degré auquel il pense être apprécié par eux.

8. Nombre de liens réciproques: score donnant le nombre de fois où un élève apprécie fortement un de ses camarades, et où cette appréciation est réciproque (le camarade l'apprécie également fortement).

9. Nombre de rejets réciproques: score identique au précédent, mais pour des rejets mutuels.

Sur la base de ces indices, nous avons également réparti les élèves selon la typologie proposée par Coie et Dodge $(1983,1988)$ afin de pouvoir tenir compte simultanément du statut sociométrique moyen et de la nature des relations (sympathie forte, moyenne, voire nulle) pour chaque sujet et ainsi regrouper les élèves selon les cinq types distincts. En effet, avec un même statut sociométrique, un élève peut par exemple être soit apprécié moyennement par ses pairs, soit fortement apprécié par certains et fortement rejetés par d'autres. Pour réaliser cette catégorisation, nous avons transformé les variables liens réciproques (LR) et rejets réciproques (RR) en écarts-réduits à l'intérieur de chaque classe afin de standardiser les données et rendre comparables les scores pour des effectifs différents. Dans un deuxième temps, nous avons calculé deux nouvelles variables (étapes intermédiaires): la préférence sociale ( $P S=Z_{L R}-Z_{R R}$ ) et l'impact social ( $I S=Z_{L R}+Z_{R R}$ ). La répartition des élèves dans la typologie s'est faite selon les critères présentés dans le tableau 1.

Tableau 1

Critères d'inclusion de la typologie (selon Coie et Dodge, 1988)

\begin{tabular}{|l|c|c|c|c|}
\hline \multicolumn{1}{|c|}{ Type } & PS & IS & $\mathbf{Z}_{\mathbf{L R}}$ & $\mathbf{Z}_{\mathbf{R R}}$ \\
\hline Apprécié & $>1$ & & $>0$ & $<0$ \\
\hline Rejeté & $<-1$ & & $<0$ & $>0$ \\
\hline Négligé & & $<-1$ & $<0$ & $<0$ \\
\hline Controversé & & $>1$ & $>0$ & $>0$ \\
\hline Moyen & $-1<\mathrm{PS}<1$ & $-1<\mathrm{IS}<1$ & & \\
\hline
\end{tabular}

\section{Résultats}

Avec l'utilisation du questionnaire Reflet, nous disposons de deux scores correspondant aux statuts sociométriques réel et imaginé. La corrélation calculée entre ces deux variables nous donne un coefficient relativement fort $(\mathrm{r}=0,62)^{1}$. Ce lien indique donc que les élèves sont à même de déterminer, avec une certaine précision, leur statut au sein de leur classe. En examinant leur degré de lucidité, on note une différence moyenne de 0,71 entre les cotes imaginées par les sujets et celles effectivement données, les scores se situant entre 0,13 et 1,94. Aucune différence ne peut être mise en évidence selon le sexe de l'élève.

1 Pour les coefficients de corrélation, nous n'indiquons pas volontairement la probabilité du test inférentiel. En effet, avec un échantillon de 263 sujets, les corrélations sont significatives (à $5 \%$ ) à partir d'une valeur de 0,12 , corrélation considérée comme faible (Cohen, 1977). 
L'analyse des résultats issus du questionnaire d'estime de soi fournit tout d'abord des indications quant aux liens entre les différentes sous-échelles. La matrice des corrélations est très similaire à celle que fournissent les auteurs de la validation de l'outil, avec cependant des valeurs légèrement plus élevées en moyenne. Les corrélations s'échelonnent en effet de 0,01 à 0,70 (moyenne de 0,35), alors que chez Pierrehumbert, Plancherel et Jankech-Caretta (1987), on les trouve entre 0,12 à 0,61 (moyenne de 0,26 ). Les différences significatives observées selon le sexe correspondent également; les garçons ayant des valeurs plus élevées pour les domaines «physique» et «apparence» $\left(\mathrm{t}_{(261)}=4,41 ; \mathrm{p}<1 \%\right.$ et $\left.\mathrm{t}_{(261)}=3,73 ; \mathrm{p}<1 \%\right)$, les filles dans le domaine «comportement» $\left(\mathrm{t}_{(261)}=-2,67 ; \mathrm{p}<1 \%\right)$.

Selon l'objectif principal de cette recherche, nous nous sommes particulièrement intéressé à analyser les liens entre les échelles d'estime de soi et les deux statuts sociométriques des élèves. Le tableau 2 reprend les différents coefficients de corrélation obtenus. Nous avons également rajouté dans ce tableau la variable «auto-évaluation». Dans le questionnaire de base, nous avons demandé à chaque élève de s'auto-évaluer (autrement dit de se donner une cote de sympathie, comme il le fait pour ses camarades dans le questionnaire sociométrique de base). Notons à ce sujet que la corrélation entre cette variable et le statut sociométrique réel n'est pas très élevée $(r=0,22)$, de même qu'avec le statut sociométrique imaginé $(\mathrm{r}=0,28)$.

Tableau 2

Corrélations entre les différents domaines de l'estime de soi et le statut sociométrique

\begin{tabular}{|l|c|c|c|}
\hline \multirow{2}{*}{\multicolumn{1}{c|}{ Estime de soi }} & \multicolumn{2}{c|}{ Statut sociométrique } & \multirow{2}{*}{ Auto-évaluation } \\
\cline { 2 - 4 } & Réel & Imaginé & 0,37 \\
\hline Social & 0,55 & 0,61 & 0,43 \\
\hline Valeur & 0,34 & 0,39 & 0,41 \\
\hline Apparence & 0,25 & 0,35 & 0,29 \\
\hline Physique & 0,23 & 0,33 & 0,19 \\
\hline Comportement & 0,16 & 0,25 & 0,16 \\
\hline École & 0,13 & 0,22 & 0,31 \\
\hline Moyenne & 0,28 & 0,36 & \\
\hline
\end{tabular}

On peut constater que l'estime de soi corrèle de manière légèrement plus forte avec le statut sociométrique imaginé que réel (différence toutefois non significative entre les corrélations). On relève particulièrement des coefficients élevés avec le domaine social, ce qui est tout à fait cohérent avec le caractère relationnel du questionnaire sociométrique. En ce qui concerne l'auto-évaluation, la prédiction moyenne est également relativement comparable aux deux autres, mais l'accent touche plus particulièrement ici le sentiment de valeur propre $(\mathrm{r}=0,43)$. Ce résultat souligne donc, de manière logique, le trait plus général de cette auto-évaluation.

En ce qui concerne la typologie des élèves, nous nous sommes intéressé à la mettre en relation avec la présence (ou non) d'une sympathie réciproque dans la classe (tableau 3). Si plus du trois-quart des élèves ont dans leur classe au moins un camarade avec lequel le lien est réciproque (avec une moyenne de 2,25 liens réciproques), certains types d'élèves en ont proportionnellement moins que les autres (les négligés et les rejetés). Vu sous un angle différent, on peut tout de même 
souligner que près d'un élève sur deux issu de ces deux catégories possède tout de même des sympathies mutuelles au sein de sa classe.

Tableau 3

Typologie d'élèves et liens réciproques dans la classe

\begin{tabular}{|l|c|c|c|}
\hline \multirow{2}{*}{} & \multicolumn{2}{|c|}{ Lien réciproque } & \multirow{2}{*}{ Total } \\
\cline { 2 - 4 } & Aucun & Un ou plus & 71 \\
\hline Apprécié & 6 & 65 & 51 \\
\hline Rejeté & 30 & 21 & 32 \\
\hline Négligé & 13 & 19 & 8 \\
\hline Controversé & 0 & 8 & 101 \\
\hline Moyen & 9 & 92 & 263 \\
\hline Total & 58 & 205 & \\
\hline
\end{tabular}

Afin d'examiner si les liens observés entre le statut sociométrique de l'élève et son estime de soi se traduisent également par des différences dans la typologie d'élèves, nous avons calculé les scores de l'estime de soi dans la dimension sociale (puisque les liens sont les plus importants dans ce domaine) pour chacun des types (figure 1). Les graphiques des scores relatifs aux autres domaines sont tout à fait similaires. On constate donc de nettes différences entre les cinq catégories. Dans cette figure apparaissent aussi les différences d'estime selon la présence ou l'absence de sympathies réciproques dans la classe.

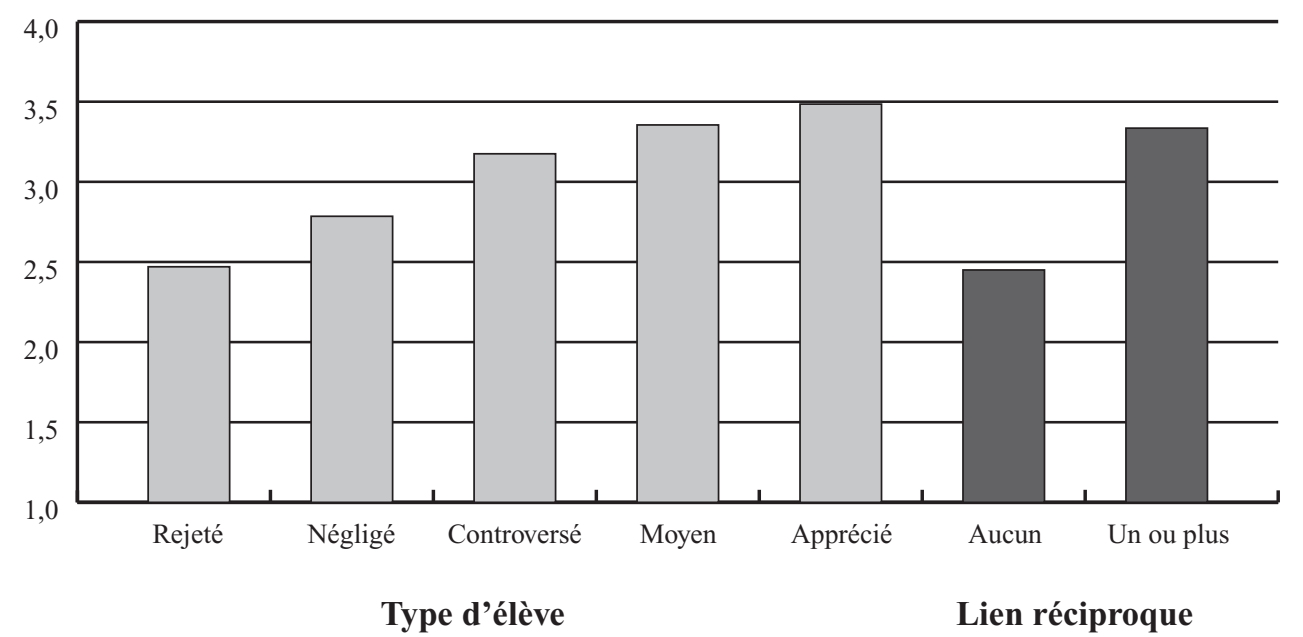

Figure 1 - Estime de soi (social) selon le type d'élève et la présence de lien(s) réciproque(s)

Les analyses inférentielles effectuées sur la base de ces données donnent des résultats significatifs tant pour la typologie $\left(\mathrm{F}_{(4,258)}=16,6 ; \mathrm{p}<1 \%\right)$ que pour la présence vs l'absence de lien réciproque $\left(\mathrm{t}_{(261)}=7,64 ; \mathrm{p}<1 \%\right)$. Les relations dans la classe sont donc clairement liées à l'estime de soi de l'élève. Constatant que plus de $10 \%$ des sujets de notre échantillon possèdent 5 liens réciproques ou plus, nous nous sommes demandé s'il y a un effet cumulatif de ce nombre de liens réciproques sur l'estime de soi et nous avons donc effectué une analyse de variance prenant en compte, comme facteur, le nombre de liens (de 0 à 5 et plus). Les résultats s'avèrent significatifs 
$\left(\mathrm{F}_{(5,257)}=14,8 ; \mathrm{p}<1 \%\right)$. En examinant la moyenne d'estime de soi pour chaque groupe, on constate les écarts les plus importants (tests post hoc de Tuckey significatifs) entre les sujets sans liens réciproques et les autres (ayant un ou plusieurs liens réciproques), soulignant ainsi l'estime de soi nettement plus faible des élèves pour lesquels aucune sympathie mutuelle avec un camarade n'est présente. Par ailleurs, il existe bien un effet cumulatif occasionné par l'augmentation du nombre de liens réciproques repérés puisque plus les élèves ont de liens réciproques, meilleure est leur estime d'eux-mêmes. La corrélation entre ces variables s'élève à $r=0,46$, mais chute à 0,15 lorsque l'on contrôle le statut sociométrique réel (corrélation partielle).

Pour améliorer la compréhension des liens entre les différents indices issus des questionnaires sociométriques, nous avons mené différentes analyses exploratoires, tout particulièrement avec l'expansivité affective de l'élève. Dans les analyses effectuées, on constate que les élèves apprécient d'autant plus un pair qu'il est jugé comme étant expansif. Le coefficient de corrélation calculé entre le statut sociométrique réel de l'élève et l'expansivité affective perçue (qui lui est attribuée par ses pairs) s'élève à 0,84 . Même en contrôlant (partialisant) l'expansivité affective réelle, cette corrélation reste à 0,82 , ce qui indique que les élèves fortement appréciés par leurs pairs sont perçus comme étant expansifs, et que cette relation est quasiment indépendante de l'expansivité réelle de ces élèves.

Finalement, pour répondre à notre hypothèse postulant la présence d'une contribution significative du statut sociométrique imaginé à l'explication de l'estime de soi, nous avons également partialisé l'analyse de corrélation afin de déterminer le lien entre ces deux variables tout en contrôlant la variable centrale, à savoir le statut sociométrique réel. Avec cette procédure, nous obtenons un coefficient significatif de 0,36 . Notre hypothèse est donc vérifiée dans la mesure où le statut sociométrique imaginé, bien que fortement corrélé avec le statut sociométrique réel, apporte une part de variance intéressante au score d'estime de soi des élèves.

\section{Discussion et conclusion}

L'objectif de cette recherche visait, dans une perspective principalement exploratoire, la compréhension des liens entre les mesures sociométriques et l'estime de soi. Plusieurs recherches antérieures, dont celle de Rudolph, Hammen et Burge (1995), se sont intéressées à articuler la perception que l'élève a de lui-même relativement à son statut social dans la classe. Dans cette étude, nous nous inspirons donc de divers travaux déjà publiés en ajoutant une nouvelle composante dans les analyses, élément rendu possible à l'aide du questionnaire sociométrique Reflet qui vise à comprendre la perception qu'a l'élève de son statut par rapport à ses camarades.

Tout d'abord, nos résultats amènent, d'une certaine manière, des arguments à la validité convergente entre le questionnaire d'estime de soi et les mesures sociométriques. On soulignera plus particulièrement une corrélation élevée entre le domaine social et le statut sociométrique imaginé ainsi que, avec un coefficient un peu moins important, le lien entre la valeur propre de l'élève et son auto-évaluation.

Les analyses effectuées nous indiquent ensuite que les élèves interrogés sont, en moyenne, relativement lucides quant à leur statut sociométrique dans la classe. Si cet aspect est plutôt intéressant pour un fonctionnement sain des relations dans le groupe, nous constatons cependant que 
près d'un quart d'entre eux n'ont pas de camarade pour lequel le lien de sympathie est réciproque. Comparativement, la recherche de Bishop et Inderbitzen (1995), réalisée aux États-Unis avec un échantillon d'âge comparable, montre un taux deux fois plus faible sur cet indicateur. L'effectif des classes de notre recherche (en moyenne 20,2 élèves par classe, avec un minimum de 14 élèves) ne semble pas être un facteur entravant fortement la création de liens mutuels. En effet, les éventualités de trouver au moins un camarade sympathique dans la classe et que cette sympathie soit réciproque reste relativement élevée. D'ailleurs, ce ne sont pas forcément dans les plus petites classes que l'on trouve en moyenne le moins de liens mutuels par élève, et bon nombre d'entre eux en comptent d'ailleurs plusieurs. Notons toutefois que la comparaison avec d'autres études peut être biaisée dans la mesure où la majorité des recherches utilisent encore une nomination de trois pairs appréciés et de trois autres rejetés, alors que nous proposons une évaluation de chaque camarade (échelles d'estimation). Les deux démarches entravent quelque peu la spontanéité des réponses, mais pas forcément de la même façon.

Le choix de la méthode a donc une influence non négligeable sur les résultats et, par conséquent, sur les conclusions qu'on peut en tirer. Ceci apparaît également dans la mise en évidence d'un effet cumulatif du nombre de liens réciproques dans la classe sur l'estime de soi, hypothèse - défendue entre autres par Asher et Parker (1989) - qui n'a pas pu trouver confirmation dans la publication de Bishop et Inderbitzen (1995) où les choix réciproques ne pouvaient être très nombreux compte tenu de la procédure utilisée (nombre de nominations restreint à trois).

Dans l'analyse des liens entre le statut sociométrique de l'élève et son estime de soi, nous obtenons des corrélations significatives. La manière dont l'élève se perçoit est donc étroitement liée à sa position sociale dans la classe. En examinant les données sous l'angle de la typologie, nous trouvons une confirmation de ce résultat. Les sujets considérés comme rejetés - faisant l'objet d'une attention particulière dans la recherche de Boivin et Begin (1989) - éprouvent en moyenne une estime de soi plus faible que leurs pairs négligés ou controversés, ces derniers obtenant d'ailleurs des scores moindres que les élèves des types moyens et appréciés. L'utilisation de la typologie s'avère donc particulièrement pertinente dans la mesure où elle est révélatrice du fonctionnement relationnel de l'élève au sein de sa classe (c'est-à-dire de manière contextualisée) et permet une comparaison fine des scores d'estime de soi.

Avec l'utilisation du questionnaire sociométrique Reflet, nous apportons un élément supplémentaire dans la compréhension du regard que l'élève porte sur lui-même. En effet, le statut social dans la classe influence sans conteste l'estime de soi de l'élève. Cependant, dans notre étude, la façon dont celui-ci imagine être évalué par ses pairs (mesuré par le statut sociométrique imaginé) semble être plus déterminante que le nombre d'amis (liens réciproques) dans la prédiction de l'estime de soi relative au domaine social. Si les différents facteurs sont forcément corrélés entre eux, on peut toutefois distinguer des liens plus importants, voire indépendants, avec l'estime de soi.

Enfin, si cette recherche n'a pas inclus l'observation des comportements des élèves, elle apporte tout de même des éléments de compréhension à la dynamique des relations dans la classe. L'expansivité affective perçue d'un élève (autrement dit s'il est perçu comme appréciant ses pairs) est fortement corrélée avec son statut sociométrique, et ceci indépendamment de l'expansivité affective réelle (ce qu'il pense réellement de ses camarades). Même si la causalité de cette relation peut être discutée, on comprend bien toutefois l'importance des perceptions dans la construction de la relation à autrui et de la relation à soi-même. 


\section{Références}

André, C. et Lelord, F. (2007). L'estime de soi. S'aimer pour mieux vivre avec les autres (3 éd.). Paris: Odile Jacob.

Asher, S.R. et Dodge, K.A. (1986). Identifying children who are rejected by their peers. Developmental Psychology, 22(4), 444-449.

Asher, S.R. et Parker, J.G. (1989). Significance of peer relationship problems in childhood. In B.H. Schneider, G. Attili, J. Nadel et R.P. Weissberg (dir.), Social competence in developmental perspective (p. 5-23). Boston, MA: Kluwer.

Babad, E. (2001). On the conception and measurement of popularity: More facts and some straight conclusions. Social Psychology of Education, 5(1), 3-30.

Bastin, G. (1970). Les techniques sociométriques. Paris: Presses universitaires de France.

Bell-Dolan, D.J., Foster, S.L. et Sikora, D.M. (1989). Effects of sociometric testing on children's behavior and loneliness in school. Developmental Psychology, 25(2), 306-311.

Bishop, J.A. et Inderbitzen, H.M. (1995). Peer acceptance and friendship: An investigation of their relation to selfesteem. Journal of Early Adolescence, 15(4), 476-489.

Boivin, M. et Begin, G. (1989). Peer status and self-perception among early elementary school children: The case of the rejected children. Child Development, 60(3), 591-596.

Boivin, M., Vitaro, F. et Gagnon, C. (1992). A reassessment of the Self-Perception Profile for Children: Factor structure, reliability and convergent validity of a French version among second through sixth-grade children. International Journal of Behavioral Development, 15(2), 275-290.

Bolognini, M. et Plancherel, B. (1998). Estime de soi et santé psychique: le rôle de la puberté et du support social. In M. Bolognini et Y. Prêteur (dir.), Estime de soi. Perspectives développementales (p. 85-105). Lausanne: Delachaux et Niestlé.

Brochin, H.A. et Wasik, B.H. (1992). Social problem solving among popular and unpopular children. Journal of Abnormal Child Psychology, 20(4), 377-391.

Cohen, J. (1977). Statistical power analysis for the behavioral sciences. New York, NY : Academic Press.

Coie, J.D. et Dodge, K.A. (1983). Continuities and change in children's social status : A five-year longitudinal study. Merril-Palmer Quarterly, 29(3), 261-282.

Coie, J.D. et Dodge, K.A. (1988). Multiple sources of data on social behavior and social status in the school : A crossage comparison. Child Development, 59(3), 815-829.

Coie, J.D., Dodge, K.A. et Coppotelli, H. (1982). Dimensions and types of social status: A cross-age perspective. Developmental Psychology, 18(4), 557-570.

Eisenberg, N., Pidada, S. et Liew, J. (2001). The relations of regulation and negative emotionality to Indonesian children's social functioning. Child Development, 72(6), 1747-1763.

Franz, D.Z. et Gross, A.M. (2001). Child sociometric status and parent behaviors : An observational study. Behavior Modification, 25(1), 3-20.

Frederickson, N.L. et Furnham, A.F. (1998). Sociometric-status-group classification of mainstreamed children who have moderate learning difficulties: An investigation of personal and environmental factors. Journal of Educational Psychology, 90(4), 772-783.

Gilly, M. (1999). Les représentations sociales dans le champ éducatif. In D. Jodelet (dir.), Les représentations sociales (p. 383-406). Paris: Presses universitaires de France.

Harter, S. (1982). The Perceived Competence Scale for Children. Child Development, 53(1), 87-97.

Harter, S. (1998). Comprendre l'estime de soi de l'enfant et de l'adolescent. Considérations historiques, théoriques et méthodologiques. In M. Bolognini et Y. Prêteur (dir.), Estime de soi. Perspectives développementales (p. 57-81). Lausanne: Delachaux et Niestlé.

Hayvren, M. et Hymel, S. (1984). Ethical issues in sociometric testing: Impact of sociometric measures on interaction behavior. Developmental Psychology, 20(5), 844-849.

Hubbard, J.A. (2001). Emotion expression processes in children's peer interaction: The role of peer rejection, aggression and gender. Child Development, 72(5), 1426-1438.

Košir, K. et Pečjak, S. (2005). Sociometry as a method for investigating peer relationship: What does it actually measure? Educational Research, 47(1), 127-144. 
LaFontana, K.M. et Cillessen, A.H. (1999). Children's interpersonal perceptions as a function of sociometric and peer-perceived popularity. Jounal of Genetic Psychology, 160(2), 225-242.

L'Ecuyer, R. (1994). Le développement du concept de soi de l'enfance à la vieillesse. Montréal: Les Presses de l'Université de Montréal.

McArdle, P., O’Brien, G., Macmillan, A. et Kolvin, I. (2000). The peer relations of disruptive children with reference to hyperactivity and conduct disorder. European Child and Adolescent Psychiatry, 9(2), 91-99.

Moreno, J.L. (1945). Sociometric measurement of social configurations : Based on deviation from chance. New York, NY: Beacon House.

Moreno, J.L. (1970). Fondements de la sociométrie (2éd.). Paris: Presses universitaires de France.

Newcomb, A.F., Bukowski, W.M. et Pattee, L. (1993). Children's peer relations: A meta-analytic review of popular, rejected, neglected, controversial and average sociometric status. Psychological Bulletin, 113(1), 99-128.

Northway, M.L. (1964). Initiation à la sociométrie (trad. par H. Jidouard). Paris: Dunod.

Ollendick, T.H., Weist, M.D., Borden, M.C. et Greene, R.W. (1992). Sociometric status and academic, behavioral and psychological adjustment: A five-year longitudinal study. Journal of Consulting and Clinical Psychology, 60(1), 80-87.

Parkhurst, J.T. et Hopmeyer, A. (1998). Sociometric popularity and peer-perceived popularity: Two distinct dimensions of peer status. Journal of Early Adolescence, 18(2), 125-144.

Pierrehumbert, B., Plancherel, B. et Jankech-Caretta, C. (1987). Image de soi et perception des compétences propres chez l'enfant. Revue de Psychologie appliquée, 37(4), 359-377.

Putallaz, M. (1983). Predicting children's sociometric status from their behavior. Child Development, 54(6), 1417-1426.

Remmers, H.H. (1963). Rating methods in research on teaching. In N.L. Gage (dir.), Handbook of research on teaching (p. 329-378). Chicago, Il: Rand McNally.

Rosenberg, M., Schooler, C. et Schoenbach, C. (1989). Self-esteem and adolescent problems : Modeling reciprocal effects. American Sociological Review, 54(6), 1004-1018.

Rudolph, K.D., Hammen, C. et Burge, D. (1995). Cognitive representations of self, family and peers in school-age children: Links with social competence and sociometric status. Child Development, 66(5), 1385-1402.

Tagiuri, R. (1952). Relational analysis: An extension of sociometric method with emphasis upon social perception. Sociometry, 15, 91-104.

Tagiuri, R., Kogan, N. et Bruner, J.S. (1955). The transparency of interpersonal choice. Sociometry, 18(4), 624-635.

Toesca, Y. (1972). La sociométrie à l'école primaire. Paris: ESF.

Worth Gavin, D. et Herry, Y. (1996). The French Self-Perception Profile for Children: Score validity and reliability. Educational and Psychological Measurement, 56(4), 678-700. 